Background In spite of recent improvements in the outcomes of preterm infants, sepsis remains to be an important cause of mortality and morbidity. Studies have noted the rise of Gramnegative infections as causes of sepsis in the preterm population.

Objectives We aim to describe the epidemiology, trends and microbial distribution of neonatal sepsis episodes among preterm infants born $<32$ weeks gestation over a 13-year period.

Methods This is a retrospective single-centre cohort study of culture-confirmed neonatal sepsis episodes, which is defined as the isolation of pathogenic organism from blood and/or cerebrospinal fluid cultures. We included newborns that were admitted to the Neonatal Intensive Care Unit (NICU) who were $<32$ weeks birth gestation and birthweight of $\leq 1500 \mathrm{~g}$. Neonatal sepsis episodes were stratified into early-onset sepsis (EOS) occurring at $<72$ hours of birth and late-onset sepsis (LOS) occurring $>72$ hours after birth up till discharge.

Results A total of 2019 preterm infants were included in the study. The characteristics of the cohort: $52.7 \%$ male, median birth gestational age 28 weeks, $19.9 \%$ small-for-gestational age, $89.9 \%$ received antenatal steroids, $85.5 \%$ had a central venous catheter. Over the study period, 263 infants had a total of 273 episodes of culture-confirmed sepsis -49 EOS and 224 LOS episodes. Incidence of sepsis in this cohort decreased from a high of 24.2\% in 2006 to 7.4\% in 2017. This was concordant with a decrease in LOS over the same period: $16.8 \%-21.6 \%$ from $2005-2006$ to $4.9 \%$ in 2017 . EOS incidence remained relatively stable, ranging from 04.4\%. Gram negative bacteria were the predominant pathogen group isolated, accounting for $186 / 281(66.2 \%)$ of all organisms- $40 / 49(81.6 \%)$ of EOS and 146/224 (65.2\%) of LOS episodes. E Coli [24/49 (49.0\%)] and Klebsiella spp [39/224 $(17.4 \%)]$ were the most common microbial causes of EOS and LOS episodes respectively. Coagulase negative staphylococcus (CONS) accounted for 36/224 (16.1\%) of LOS. Fungal infections were predominantly due to Candida spp - 12.1\% (27/ 224 ) of LOS. Of note, there were no fungal infections detected over the recent 5 years.

Conclusions The decrease in the overall incidence of neonatal sepsis among preterm infants $<32$ weeks in our centre over the 13-year study period, is due to a reduction in LOS. Gram-negative bacterial organisms are the predominant infecting organism in this population with E.Coli and Klebsiella spp being the most common causes of EOS and LOS.

\section{KNOWLEDGE, ATTITUDES AND EXPECTATIONS OF PERINATAL CARE DURING THE COVID-19 PANDEMIC}

Samantha Goh, Caleb Lim, Juin Yee Kong, Kee Thai Yeo, Manisha Mathur, Ka-Hee Chua. Singapore

10.1136/bmjpo-2021-RCPCH.7

Background There is limited evidence regarding the transmission SARS-CoV-2 from mother to infant, as well as the optimal management of infected women and infant during pregnancy, labor and early postnatal period. The knowledge, concerns and expectations of women with regards to perinatal and neonatal care during this current COVID-19 pandemic is currently unclear.

Objectives This study aimed to investigate the knowledge and expectations of pregnant women on perinatal and neonatal care during the coronavirus disease 2019 (COVID-19) pandemic in Singapore.

Methods A cross-sectional survey was administered via a secure online platform to pregnant women attending the antenatal clinics between August 2020 and September 2020. Participants aged $>21$ years who were pregnant and had no history of confirmed COVID-19 were included in the study. The survey consisted of 10 questions formulated to evaluate the knowledge and expectations of these women on the perinatal and neonatal care during this current pandemic.

Results A total of 313 pregnant women completed the survey during the study period. The mean age of the participants was 30 years (SD 4; range 22-43 years). The median gestational age of women at survey participation was 25 weeks (range 4-40 weeks). The participants were predominantly multiparous (54\%) and almost all (98\%) had completed secondary level education. Majority of participants were aware of the spread of COVID-19 by respiratory secretions and contact (90\%), and the importance of hand hygiene and face masking (94\%). Up to $72 \%$ agreed or strongly agreed that in-utero transmission of SARS-CoV-2 to the unborn foetus was possible. Most were unsure of the optimal mode of delivery $(77 \%)$ and only $22 \%$ believed that breastfeeding was safe in a pregnant women with active COVID-19. There was no significant association between the sociodemographic factors evaluated and maternal agreement with the possibility of in-utero SARSCoV-2 transmission and the risk associated with vaginal delivery in women with COVID-19. Although 46\% of participants were concerned about the increased risk of contracting COVID-19 during routine clinic appointments at the hospital, only $37 \%$ of the cohort were agreeable with teleconferencing of clinic appointments. More than half $(56 \%)$ of the participants reported that their postnatal confinement plans were affected by the current pandemic.

Conclusions Our survey revealed that majority of participants were aware of modes of transmission and the prevention strategies of SARS-CoV-2, there were however significant gaps identified in their knowledge related to the risk of in-utero transmission and safety of breast feeding along with significant variability to the agreement with alterations to the perinatal care. For best practice we recommend provision of evidence based information early to expectant mothers by the healthcare professionals to reduce misinformation and anxiety amongst pregnant women related to the current pandemic.

\section{HEALTH STATUS OF CHILDREN WITH DOWN SYNDROME IN MYANMAR}

Khine Mi Mi Ko, Khine Mi Mi Ko, Kyaw Linn, Aye Mya Min Aye, Mon Thander Saw, Pyae Phyo Aung. Myanmar

\subsection{6/bmjpo-2021-RCPCH.8}

Background Down Syndrome children have complex healthcare needs. There was no previous data of Pediatric Down Syndrome (PDS) in Myanmar and little is known about their health status and challenges to seek proper screening tests and health care services. Since there are no established local guidelines for PDS, limited resources and inaccessibility to specialty centers especially for those form remote areas, more health care challenges are encountered throughout the country.

Objectives To assess the health status, screening tests and follow up conditions of Myanmar Down Syndrome children 
Methods One hundred and sixty six children with Down Syndrome less than 16 years were collected from regional hospitals within one year (January to December 2020). We took history, reviewed medical records and performed clinical examination. We analyzed demographic characteristics, attitude and healthcare knowledge of caregivers, screening tests and following up conditions including problems in coping of caring the children with Down Syndrome.

Results Results: Of the 166 participants, majority, (53\%) were male. A total of 95(57.2\%) were from urban area and most of PDS (96.4\%) were cared by biological parents. Half of caregivers in this study group were nongraduate (most are middle-school education). Although (80.7\%) were born at regional hospitals, some were unaware of Down Syndrome until the child developed clinical symptoms (maximum age up to 9 years) and (45\%) have lack of knowledge about associated health problems. The most common medical conditions reported were cardiac (53.01\%), thyroid (13.25\%), visual (13.25\%), hearing problems (8.43\%).Screening tests done for cardiac, thyroid, vision and hearing were $72.89 \%, 40.96 \%$, $25.3 \%$ and $26.51 \%$ respectively and only $(21.38 \%)$ have regular follow up after diagnosis. Most of the caregivers have lack of knowledge about how to care their children, importance of screening tests according to age and regular follow up. Conditions such as financial difficulties, social problems and uneasily accessible health specialties especially for those who live in rural areas became barriers to receive proper health care services.

Conclusions More data are needed to understand health conditions, caregiver knowledge and explore the barriers in seeking proper health care services. Appropriate policies, feasible local guidelines and more education programmes to address health care challenges for Down Syndrome children are crucial to raise awareness of healthcare personals and reduce the gap to receive proper healthcare services. Screening tests according to age and long-term care programs are vastly underdeveloped in poor countries like Myanmar, it will be essential for complementing the support system and sustaining the major role that families or caregivers currently play.

\section{THE RELATIONSHIP OF LINGUAL FRENULUM AND OBSTRUCTIVE SLEEP APNOEA (OSA) IN CHILDREN}

Hoi Man Yuen, Chun Ting Au, Albert Martin Li, Kate Ching Ching Chan. Hong Kong

\subsection{6/bmjpo-2021-RCPCH.9}

Background Childhood obstructive sleep apnoea (OSA) is a prevalent disease, and reported to affect around 5\% of primary school-aged children. It is also clinically important as it can lead to neurocognitive, metabolic and cardiovascular complications. The tongue plays an important role in maintaining patency of the oropharynx. Lingual frenulum, a connective tissue between the floor of the mouth and the underside of the tongue, can affect the tongue position, its elevation and movements. Short lingual frenulum has been reported to be a risk factor for OSA. How frenulum length and its mobility affects craniofacial development and morphology remains to be defined.

Objectives In this study we aimed to prospectively evaluate the lingual frenulum length by free tongue measurement and tongue mobility in children suspected to have OSA. We hypothesized that OSA children would have shorter lingual frenulum than their non-OSA counterparts. Moreover, we explored the relationship between frenulum length and craniofacial profile using cephalometry. We hypothesized that cephalometric measurements would be different in children with and without short frenulum.

Methods Prepubertal Chinese children aged 5-12 years old, suspected to have OSA were recruited. Anthropometric measurements including weight, height, and circumferences of waist, hip and neck were taken on the day of admission. The lingual frenulum was evaluated based on tongue mobility and free tongue length. Tongue mobility obtained by a digital calliper was defined by Mpal/Mmax, which are the maximal distances between incisors during full mouth opening (Mmax) and when the tongue tip touched the palatal papilla (Mpal). The free tongue length was measured from the insertion of the lingual frenulum to the tongue tip using Quick Tongue-tie Assessment Tool (QTT). Normal tongue mobility was defined as mobility $\geq 50 \%$, and normal free tongue length was defined as $\geq 16 \mathrm{~mm}$. Cephalometric analysis was performed to evaluate the craniofacial profile. OSA was defined as obstructive apnoea hypopnoea index (OAHI) $\geq 1 / \mathrm{h}$ from overnight polysomnography.

Results In this study, 86 subjects (mean age: $8.36 \pm 1.69$ years) were recruited, and 50 were diagnosed to have OSA $(\mathrm{OAHI} \geq 1 / \mathrm{h})$. There was no significant difference in anthropometric measurements between OSA and non-OSA groups. The medians of the free tongue length in OSA and non-OSA groups were 20 and $24 \mathrm{~mm}(\mathrm{p}=0.321)$, respectively. The mean tongue mobility was $0.583( \pm 0.189)$ in OSA group, and $0.680( \pm 0.152)$ in non-OSA group $(p=0.010)$. Free tongue length was significantly correlated with most of the anthropometric variables including age, weight, height, BMI, waist, and hip circumferences, but did not correlate with any of the PSG variables. Tongue mobility was not correlated with any anthropometric variables, but inversely correlated with OAHI $(\mathrm{r}=-0.234, \mathrm{p}=0.030)$. In multivariate logistic regression, tongue mobility was independently associated with the presence of OSA after adjusted for age and gender. Tongue mobility was correlated with the cranial base angle $(\mathrm{Ba}-\mathrm{S}-\mathrm{N})$, which can affect the relative position of the mandible.

Conclusions Reduced tongue mobility is associated with OSA in prepubertal children. Furthermore, tongue mobility may be an important factor in driving mandibular development.

\section{ARE WE IN CONTROL OF OUR DEMONS? UNDERSTANDING COMPASSION SATISFACTION, COMPASSION FATIGUE AND BURNOUT IN AN ASIAN PAEDIATRIC EMERGENCY IN A PANDEMIC}

Kar Yee Catrin Kong, Sashikumar Ganapathy. Singapore

\subsection{6/bmjpo-2021-RCPCH.10}

Background The medical profession, with its remarkable physical and emotional demands, predisposes physicians to compassion fatigue (CF) and burnout (BO). Though these conditions have been studied individually, little attention has been paid to how pediatric emergency physicians experience these conditions in the context of an Asian emergency setting especially during a global pandemic.

Objectives In our study, we aim to understand the experiences of individual physicians and describe the potential triggers or 